collected using quantitative technique and a structured questionnaire written in Kinyarwanda was administered and completed by 1098 men.

Findings The study findings showed that the prevalence of circumcision is $16.5 \%$ (95\% CI 14.3 to $18.7 \%$ ) in the study population. This proportion is the highest in the City of Kigali (52.5\%) and in the Western Province (25.9\%). The district of Rusizi in the western Province has the highest rate of circumcised men in our study, compared to other districts (71\%). Among $63.3 \%$ of circumcised men, the procedure was performed by a healthcare provider. An estimated $50.2 \%$ of uncircumcised men would consider being circumcised and $78.5 \%$ would support circumcision for their sons and uptake will steadily increase if services are provided. The main reason would be to prevent them to be infected with STIs (including HIV) and Hygiene. The major reasons preventing uncircumcised men to circumcise is being too old for that procedure, the fear of pain and not becoming sexually promiscuous.

Conclusion This study demonstrates that male circumcision prevalence in Rwanda remains low although $M C$ is a known and acceptable practice among the study participants. The major barriers to $\mathrm{MC}$ acceptability can be removed by effective interventions like education and social mobilisation. Interventions should also target women as they can play a key role in the decision making process about circumcision within their couples and families.

\section{P5-S2.02 ABSTRACT WITHDRAWN}

\section{P5-S2.03 PHASE 1 IMPLEMENTATION OF MALE CIRCUMCISION AS A COMPREHENSIVE PACKAGE OF HIV PREVENTION IN RWANDA}

doi:10.1136/sextrans-2011-050108.535

\section{J Mbabazi. TRACPlus, Kigali, Rwanda}

Background Rwanda adopted Male Circumcision (MC) as part of a comprehensive package of HIV prevention strategies since 2007. In order to facilitate national roll out of $\mathrm{MC}$, a Phase 1 campaign involving training of healthcare providers in Nyanza and Ruhengeri District Hospitals and their affiliated health centers targeting adolescents and adults was held from 30 August to 24 September 2010. The Center for Treatment and Research on AIDS, Malaria, Tuberculosis and Other Epidemics (TRAC Plus) provided MC kits and financial support (training, cost of $\mathrm{MC}$ procedure) to two selected districts hospitals (Nyanza and Ruhengeri) to implement phase 1 . The aim of this training was to accelerate the implementation of safe, affordable and accessible male circumcision as an HIV prevention strategy while contributing in strengthening the existing programs. The quality of the MC services provided through routine data collection and formative supervision was ensured.

Methods The training provided covered both theoretical and practical aspects of $\mathrm{MC}$ as an HIV prevention strategy considering the WHO training modules. The training was conducted by a team of five National trainers (three doctors, two counsellors) and the selection criteria of participants (doctors and nurses) considered those with minimum surgical skills.

Results The training was for 10 days and 25 trainees (Medical doctors and nurses) from each District Hospital and health centers were trained in both theory and practical courses on Male Circumcision as an HIV prevention strategy and also provision of counselling on $\mathrm{MC}$ to the client before surgery. A 1-day training of community health workers on community sensitisation was done.
Following the 2-week practical session in the two DH, 120 male circumcisions were performed and acceptability for HIV testing was $100 \%$. Communication messages on male circumcision as an HIV prevention strategy and the other benefits of $\mathrm{MC}$ were provided to the communities. The follow-up visit of all people circumcised was done, and no adverse events were reported.

Conclusion The MC phase 1 was successfully implemented in two district hospitals of Nyanza and Ruhengeri and the evaluation will be done. Among the lessons learnt were mainly on the coordination of $\mathrm{MC}$ activities as it requires a focal point to facilitate the activity on a continuous basis.

\section{P5-S2.04 ASSESSING COMPREHENSION OF KEY INFORMED CONSENT CONCEPTS AMONG CLIENTS UNDERGOING MALE CIRCUMCISION DURING SCALE UP OF SERVICES IN ZAMBIA AND SWAZILAND}

doi:10.1136/sextrans-2011-050108.536

B Friedland, L Apicella, M Sheehy, K D Schenk, K Munjile, A Adams, P C Hewett. Population Council, New York, USA

Background We evaluated the informed consent (IC) process for male circumcision $(\mathrm{MC})$ as services were being scaled up in Swaziland and Zambia. A primary objective was to assess male clients' comprehension of key concepts in the informed consent process to improve, standardise and streamline consent procedures as service delivery was expanded.

Methods In a post-test study design, we asked adult (18 years and older) and adolescent (13-17 years) clients who had completed counselling, but had not yet undergone $\mathrm{MC}$, to respond to a 10 question, interviewer-administered, true-false comprehension test. We also collected data on demographics, recruitment for MC, and type of counselling received (group, one-on-one, VCT), and administered literacy and numeracy tests. Participants represented a convenience sample recruited from selected sites in urban Lusaka, and urban and rural sites in Swaziland. We used T-tests to test the null hypothesis that $90 \%$ of clients could pass the test (could score $\geq 80 \%$ ), $\chi^{2}$ tests to compare scores between subgroups (eg, adults vs adolescents), and multivariate logistic regression models to explore factors associated with passing the comprehension test.

Results Between November 2009 and August 2010, 228 MC clients in Lusaka ( $\mathrm{n}=159$ adults, 69 adolescents) and $953 \mathrm{MC}$ clients in Swaziland ( $\mathrm{n}=756$ adults, 197 adolescents) participated in the comprehension assessment. Most clients (90\%, Swaziland; $89 \%$, Zambia) passed the comprehension test; however, the pass rate in Swaziland was significantly higher among adults than adolescents ( $96 \%$ and $85 \%$, respectively; $\mathrm{p}<0.001)$. The question that posed the greatest difficulty to clients was about surgical risks; $67 \%$ of Zambian clients (71\% adults; $56 \%$ adolescents) and $87 \%$ of Swazi clients (adults and adolescents) incorrectly responded that MC surgery has no risks. Factors significantly $(\mathrm{p}<0.05)$ associated with passing the test included: literacy (both countries); advanced education (Zambia); counselling language (Zambia); and age (Swaziland). In addition, not passing the test was significantly associated with hearing about MC from a friend (vs any other source) in Swaziland.

Conclusions Service providers must place greater emphasis on potential risks of $\mathrm{MC}$ surgery to ensure that clients are fully informed before they consent to the procedure. Counselling messages should be tailored for adolescent clients, who may have lower levels of education and less sexual experience than adults undergoing male circumcision. 